\title{
Numerical Simulation of Decompression Process of a Mars Rover in the Launch Phase
}

\author{
Weizhang Wang, ${ }^{1}$ Wei Rao, ${ }^{2}$ Qi Li, ${ }^{2}$ Hao Yan, ${ }^{1}$ and Rui Zhao ${ }^{1}$ \\ ${ }^{1}$ School of Aerospace Engineering, Beijing Institute of Technology, Beijing 100081, China \\ ${ }^{2}$ Beijing Institute of Spacecraft System Engineering (ISSE), Beijing 100094, China \\ Correspondence should be addressed to Rui Zhao; zr@bit.edu.cn
}

Received 29 July 2021; Accepted 24 November 2021; Published 2 February 2022

Copyright (c) 2022 Weizhang Wang et al. Exclusive Licensee Beijing Institute of Technology Press. Distributed under a Creative Commons Attribution License (CC BY 4.0).

This paper performs numerical simulation on the decompression process of a Mars rover using FLUENT. The pressure differential between the inside and outside of the Mars rover resulting from changes in ambient pressure of the rocket fairing is investigated. In terms of numerical simulation, PROFILE outlet boundary conditions are developed and the impacts of ambient pressure settings, time steps, and mesh density are investigated to improve the accuracy of simulation results. The decompression process of the separate large module, large and small modules under two types of ambient pressures are simulated. The results show that the largest pressure differential between the inside and outside of the module body is less than $2200 \mathrm{~Pa}$. Because of the small size of the small module, the results for the separate large module and the large/small modules are consistent. The pressure differential between the inside and outside of the rover is mainly influenced by the variation in ambient pressure.

\section{Introduction}

In Mars exploration mission, the rover, which contains a nonsealed module, uses heat sealing for aerodynamic thermal protection. Nevertheless, it is difficult to conduct quantitative analysis on the air permeability of the structure of the cabin. Besides, the pressure-bearing capacity of the cover on the top of the small end of the rover is limited. In the process of launch, the ambient pressure in the rocket fairing drops dramatically and the pressure differential between the inside and outside of the rover might exceed the bearing capacity of the cover. Therefore, holes are made in the low heat flow area on the surface of the rover to make sure that the pressure differential between the inside and outside in the launch phase falls within the range of the bearing capacity of the cover.

The process of decompression in the module can be reduced to the process of deflation in a container, which is a complex unsteady-state process of variation in polytropic indices and heat transfer coefficients. In the deflation process, velocity field, temperature field, and pressure field vary over time. But on the real-world engineering issues, it is usually simplified as an adiabatic deflation process or isothermal deflation process $[1,2]$. Due to the complexity of the degas- sing process, a lot of experiments were mostly carried out on the test bench to obtain systemic degassing characteristics on different parameters [3]. With an increase in the level of computer numerical simulation, Computational Fluid Dynamics (CFD) greatly reduces the amount of experimental work, but some phenomena, such as complex physical boundaries and the speed of gases reaching the speed of sound or more in the degassing process, still exist. Therefore, the calculation of the flow field inevitably involves multidimensional transient flow simulation, complex meshing and massive amounts of meshes, and other issues [4]. In addition, the degassing performance of the degassing system is influenced by many factors [5-7], such as the initial pressure in the container, the length, inner diameter, inner wall roughness of the tube, the effective bore, the length of the bore, the inner wall roughness of the bore of the automatic valve, the possibility of sudden changes to the gas path, ambient conditions, and state of gas media. Jin et al. [8-10] of Harbin Institute of Technology carried out research on the inflation/deflation processes of the empty container, put forward a method for modeling and determination of heat exchange coefficients based on experimental and theoretical analysis, verified its accuracy through 


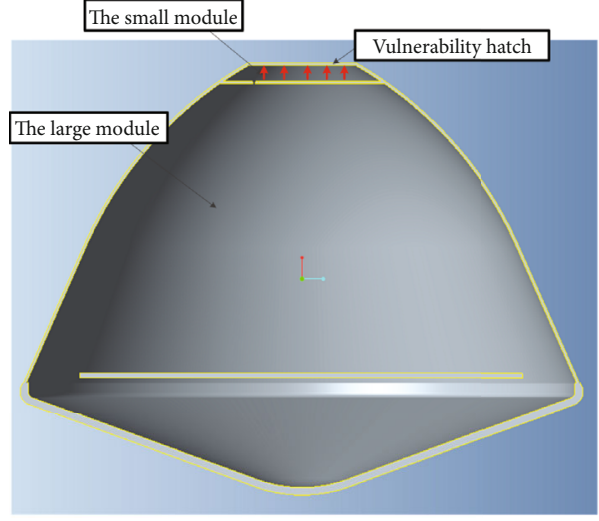

(a) The small/large module position diagram

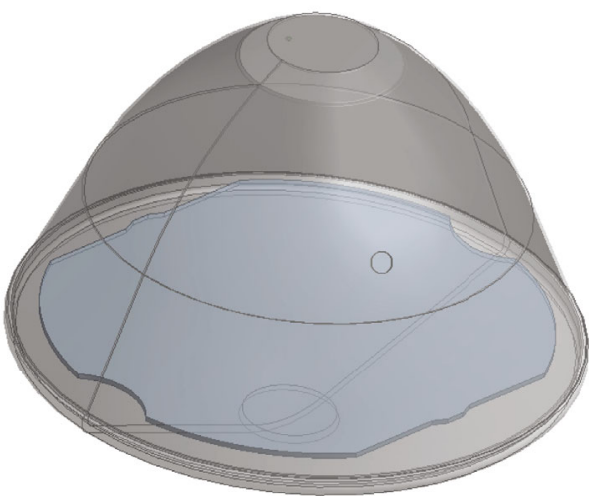

(b) Appearance of the non-sealed module

Figure 1: Model outline diagram.

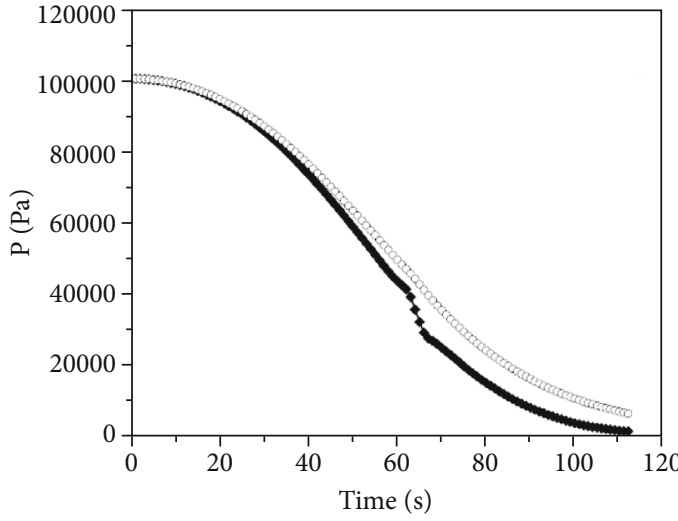
The upper limit of internal pressure

(a) Condition 1 of pressure drop

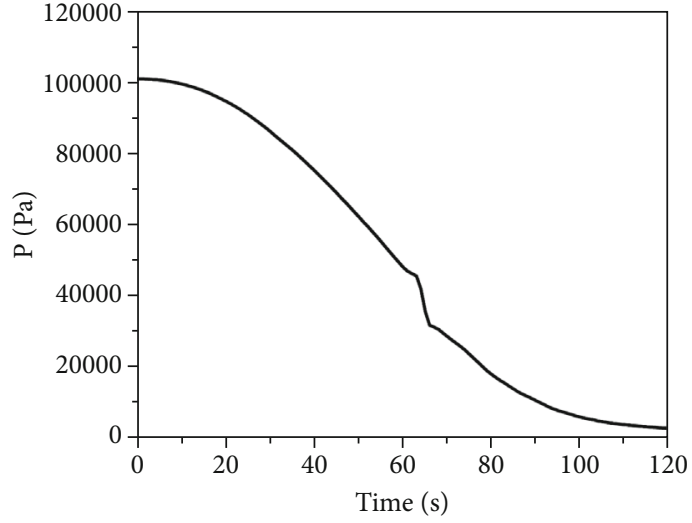

(b) Condition 2 of pressure drop

Figure 2: Conditions of pressure drop.

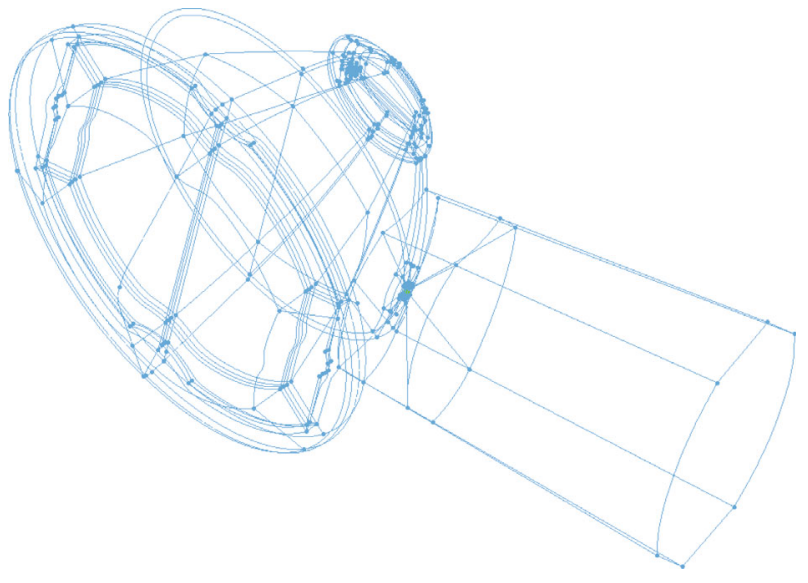

FIgURE 3: Topology of the meshes.

experiments, and discussed the heat exchange coefficient on the state parameters of the system. In addition, Li et al. [2] calculated the flow field of the aerodynamic inflation/deflation system and presented a calculation method for onedimensional unsteady flow field that takes into account fric- tion and heat transfer. However, in the abovementioned research, the simulation of the flow field in the deflation process is on pipes and the lumped parameter model used in the container does not reflect variation in distribution and variation of temperature and speed in the intrinsic deflation process of the container. Li et al. [11] of the China Academy of Engineering Physics investigated the deflation time it takes for the deflation system, which consists of a larger container, multiple segments of slender tubes of different diameters and lengths, and automatic valves, when reducing the initial pressure of $0.6 \mathrm{MPa}$ to the residual pressure of $0.001 \mathrm{MPa}$ through deflation, deduced related calculation formulas, and proposed that the whole deflation process be divided into two phases: sonic and subsonic. Li et al. [12] of the China Academy of Engineering Physics created a formula for the variation of pressure in the container based on the opening deflation system for the mechanism design of the deflation system in low-pressure environments to simplify the calculation process and shorten the calculation time. Yang et al. [13] described the process of constant volume inflation and deflation of high-pressure gases by creating mathematical equations. The results show that the inflation 




FIGURE 4: Wall surface meshes of the large module.

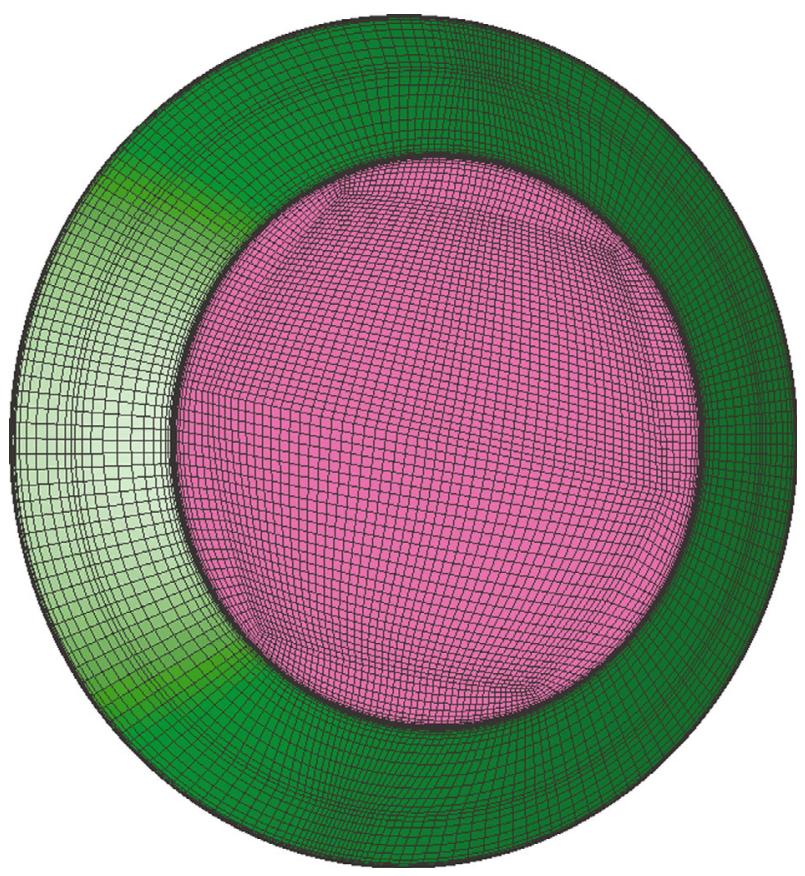

FIgURE 5: Wall surface meshes of the small module.

and deflation process of gases matches with the results of the ideal gas model. Huang et al. [14] investigated the regulation characteristics of annular-slit pressure-regulating valves. Results show that the annular-slit pressure-regulating valve can better meet the requirements of pressure regulation of transient wind tunnels. Kuptsov et al. [15] described the parameters and conditions of vertical exhaust in the critical and subcritical states and put forward two methods for calculating the draining time of the container.

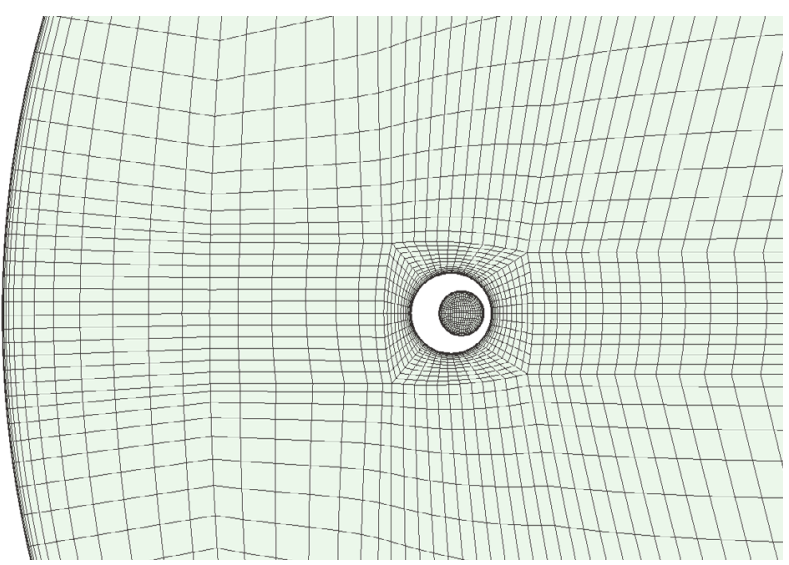

FIGURE 6: Wall surface meshes of the small hole, intermediate cable, and surrounding.

This paper simulates the decompression process of the Mars rover in the taking-off stage by means of numerical simulation. The effects of environmental pressure setting, time step, and mesh density on simulation results are studied to improve the accuracy of calculation results. The laws of variation in the pressure differential between the inside and outside of the module resulting from changes in the ambient pressure in the rocket fairing are studied.

\section{Model Design}

The subject of research in this paper is a nonsealed module. It has a maximum volume of less than $12 \mathrm{~m}^{3}$, a maximum outer diameter of $3401 \mathrm{~mm}$, and a height of $2608 \mathrm{~mm}$. The cover is on the top of the module, and the opening is located in the low heat flow area on the leeward surface of the module, with a diameter of $130 \mathrm{~mm}$. The diameter of the opening between the large module and the small module is $35 \mathrm{~mm}$. The cable that passes through the small opening is $20 \mathrm{~mm}$. This structure is simplified, with only the bodies of the large/small modules, small holes between modules, cables, openings on the large module, and baffles remained, as shown in Figure 1.

By taking the inner pressure design belt of the fairing as a condition of pressure drop in the environment outside of the module, the conditions of pressure drop corresponding to two operating conditions for the research in this paper are shown in Figures 2(a) and 2(b), respectively.

\section{Numerical Methods and Verification}

3.1. Computational Meshes and Generation Methods. Mesh generation techniques are an important part of CFD. Commercial software POINTWISE was used to generate fullstructure meshes, and the number of meshes is about 1.45 million. The computational domain consists of three parts: large module body, small module body, and radiant outer domain. To ensure the accuracy of the results, a radiant outer domain along the direction of openings is added on the outside of the opening of the large module and the boundary of the outer domain serves as the pressure 


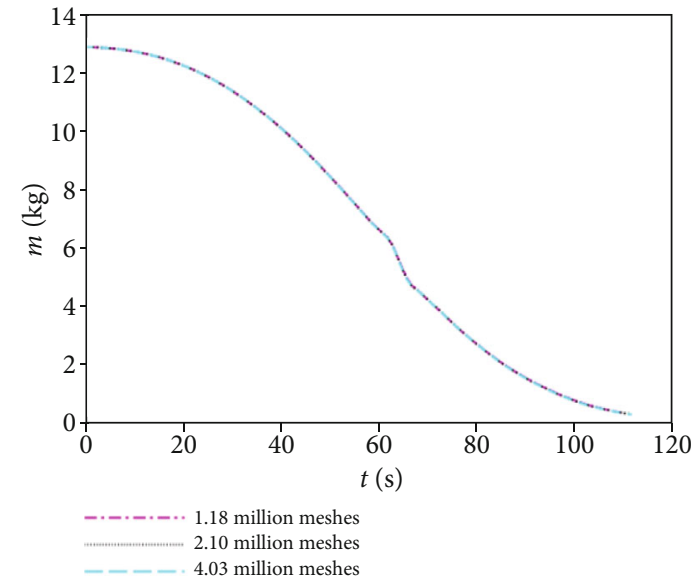

FIGURE 7: Curves of variation in the quality inside the large module.

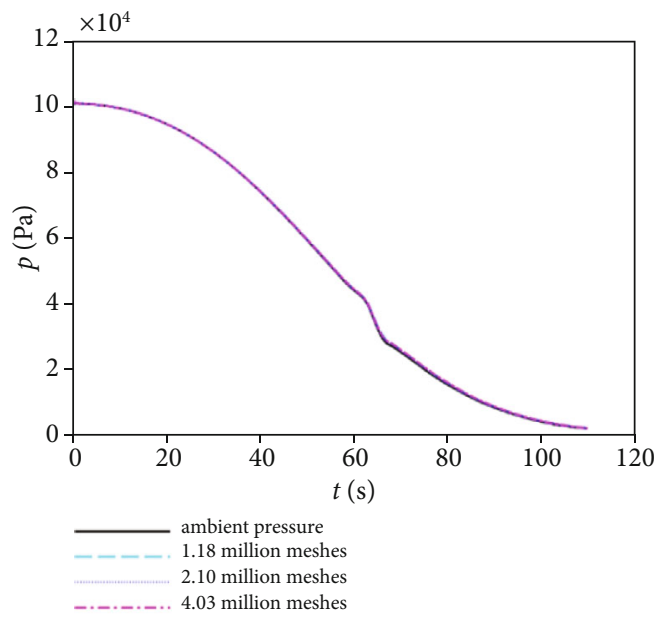

Figure 8: Curves of pressure inside the large module.

boundary condition. Figures 3-6 show the topology of the entire computational meshes, the wall surface meshes of the large/small modules, and the wall surface meshes of the small hole and in its proximity. A fluid domain of a certain size is provided on the outside of the large hole in the large module, and the boundary condition is set as pressure outlet to simulate the ambient pressure outside the module. In the deflation process, the deflation system is in a sealed environment with stable temperature. Therefore, it is assumed that the decompression process of the rover researched in this paper is an isothermal deflation process [11], the wall surface of the module body uses an isothermal wall, and the temperature of the wall surface is $300 \mathrm{~K}$.

3.2. Verification of Numerical Schemes. The fluid simulation module FLUENT in the commercial software package ANSYS is used to perform numerical simulation on the decompression process of the module. In this decompression process, the velocities in most areas are close to zero. Therefore, a pressure-based solver is used to solve problems.

In the process of numerical discretization, advection terms are discretized with a second-order upwind scheme and least-squares construction based on cells is utilized for

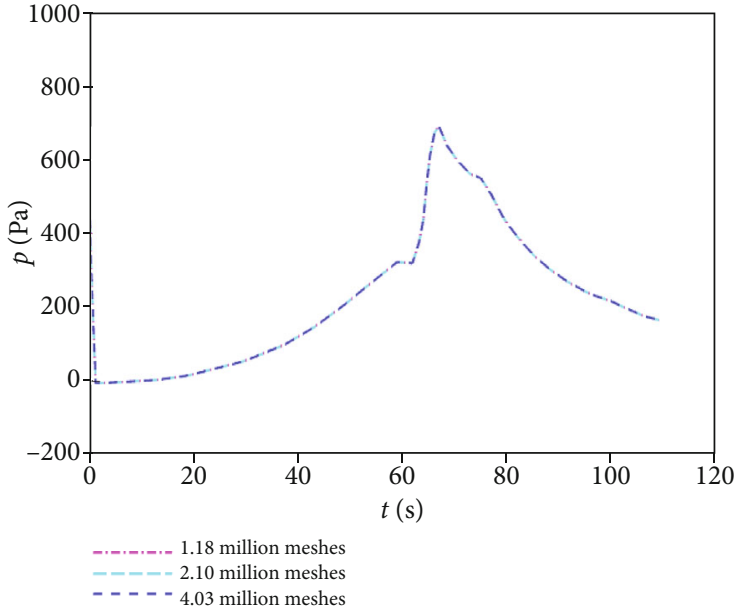

FIGURE 9: Curves of variation in pressure differential between the inside and outside of the large module.

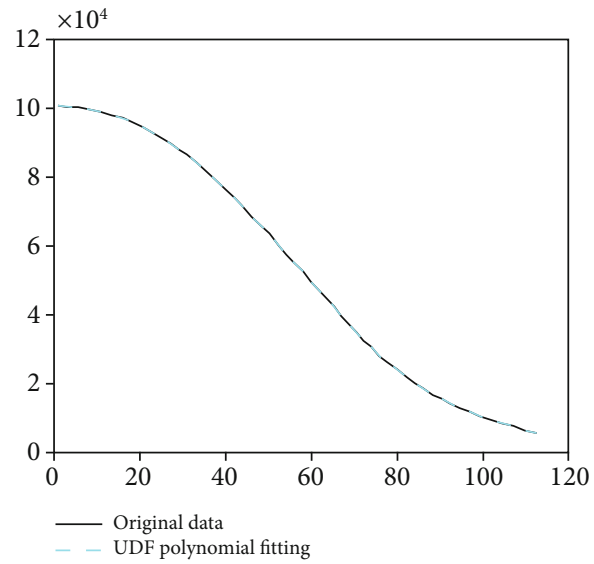

Figure 10: Polynomial fitting of ambient pressure.

diffusion terms; pressure-velocity coupling is performed using the SIMPLE algorithm; a second-order implicit scheme is used in time discretization, and the ideal gas model and realizable $k-\varepsilon$ turbulence model are applied.

There are three main factors that influence the accuracy of calculations in this paper: ambient pressure boundary settings, time scheme, and computational meshes. In this section, numerical schemes are verified in these three aspects to ensure the reliability of computation.

3.2.1. Mesh Independence. In this section, mesh independence verification is performed for the meshes of the large module with the numbers of meshes of 1.18 million, 2.10 million, and 4.03 million, respectively, to research the impact of the density of computational meshes on the results. The lower limit of internal pressure in condition 1 where the pressure changes dramatically is selected as ambient pressure, and $0.02 \mathrm{~s}$ is selected as the maximum time step.

The curves of variation in the mass and pressure inside the large module with different numbers of meshes are shown in Figures 7 and 8. The results suggest that the curves 


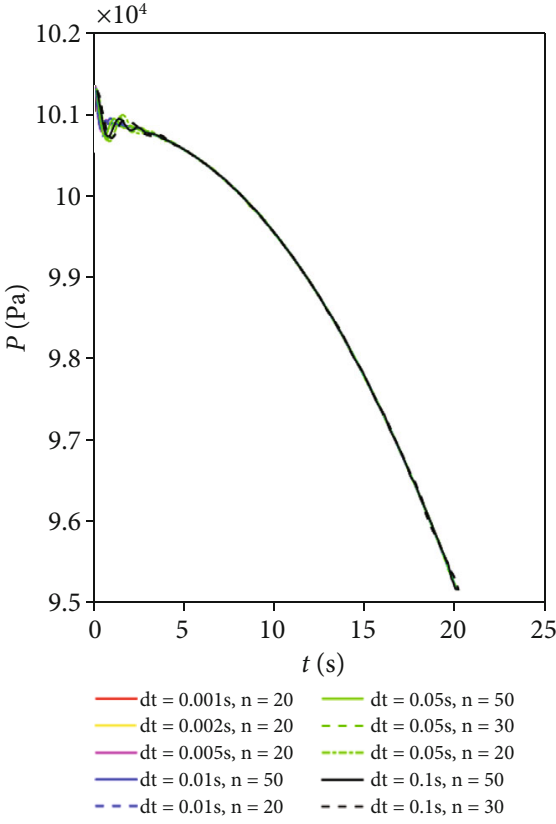

(a) Pressure in the first $20 \mathrm{~s}$

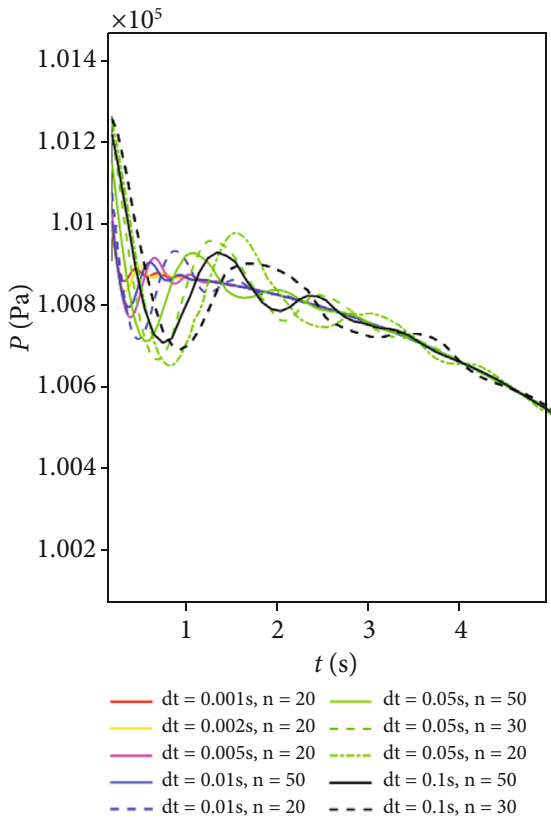

(b) Pressure in the first $5 \mathrm{~s}$

Figure 11: Comparison of different time steps and the number of iterations.

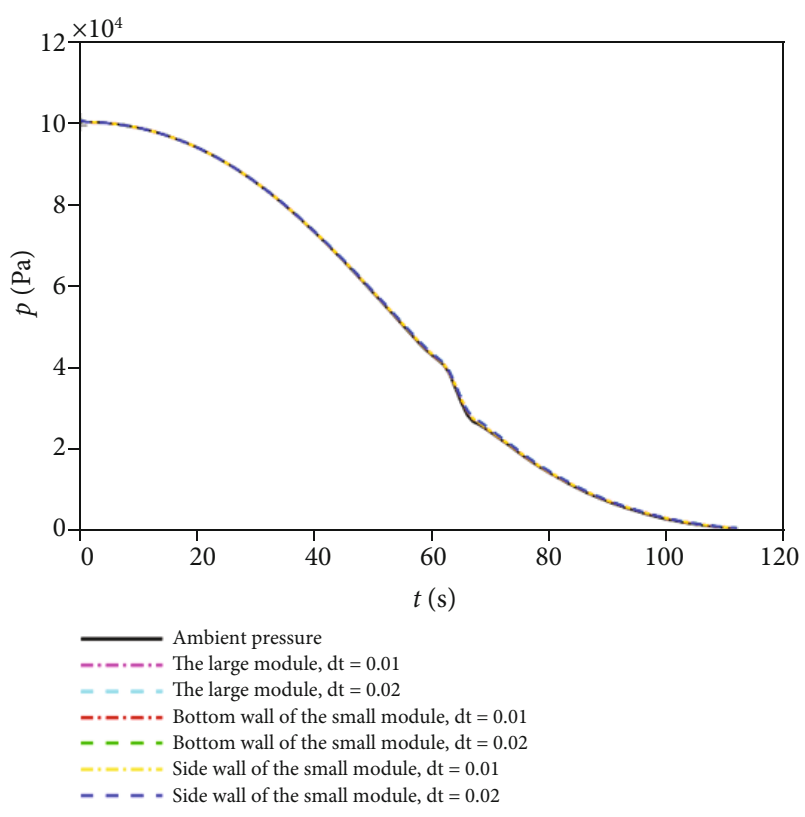

Figure 12: Curves of pressure in the large and small modules.

of variation in the mass and pressure inside the module are basically consistent despite different densities of meshes.

The curves of variation in pressure differential between the inside and outside of the module are shown in Figure 9. The results show that the laws of variation in pressure differential between the inside and outside of the module are basically the same with no obvious difference and there is only some difference in maximum values. In addition, the laws of variation in pressure differential are not monotonic with an increase in the density of meshes. When the number of meshes is 1.18 million, the respective pressure differential between the inside and the outside is the largest and the results are relatively conservative and it is also possible to save computational resources.

3.2.2. Ambient Pressure Simulation. It is crucial to simulate the ambient pressure outside the module. On the one hand, static pressure needs to be set at the boundary of the outlet for pressure outlet boundary conditions. The setting of static pressure is only used in subsonic flow. If the local flow velocity reaches the supersonic speed, the set pressure will no longer be used. Pressure is extrapolated from the inside of the flow field, and other flow parameters are also extrapolated from the inside. On the other hand, backflow conditions need to be defined for pressure outlet boundaries in favor of convergence calculation.

Change of ambient pressure outside the module is given in Figure 2 and normally defined using UDF or PROFILE in FLUENT software in calculation. This paper uses the two methods, respectively, to fit and compare the ambient pressure outside the module and discusses the results.

First, use a user-defined function (UDF) to define variation in ambient pressure. UDF uses a polynomial to fit the variation characteristics of pressure. As shown in Figure 2(a), the upper limit of internal pressure for ambient pressure 1 is easier to fit; as shown in Figure 10, the fitted curves match well with raw data when using a sixth-order polynomial to do the fittings. However, it can be seen from Figure 2 that there are sudden changes in the lower limit of internal pressure for ambient pressure 1 and ambient pressure 2, which makes it difficult to fit the variation characteristics of pressure. Therefore, UDF is not suitable to simulate the outside complex pressure environment of the Mars rover. 


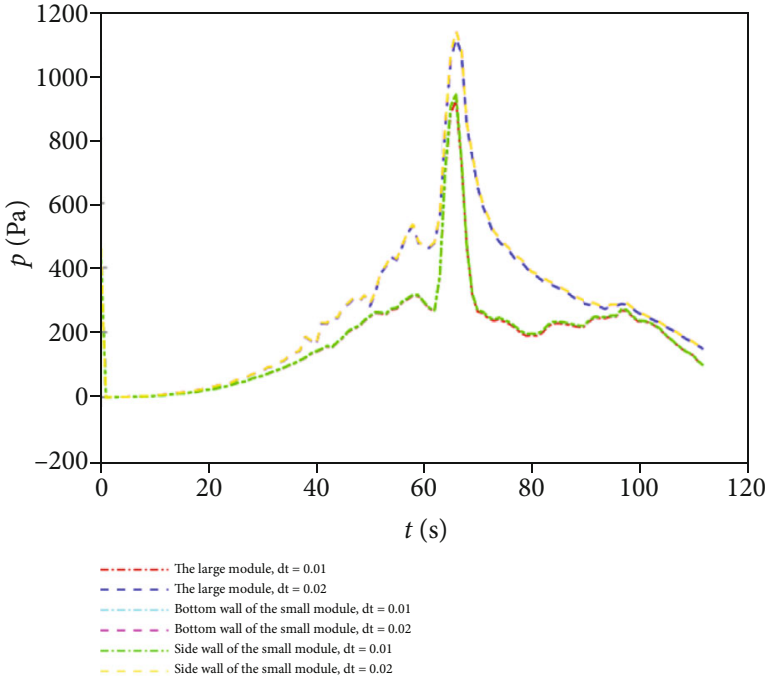

FIgURE 13: Curves of pressure differentials between the inside and outside of the large and small modules.

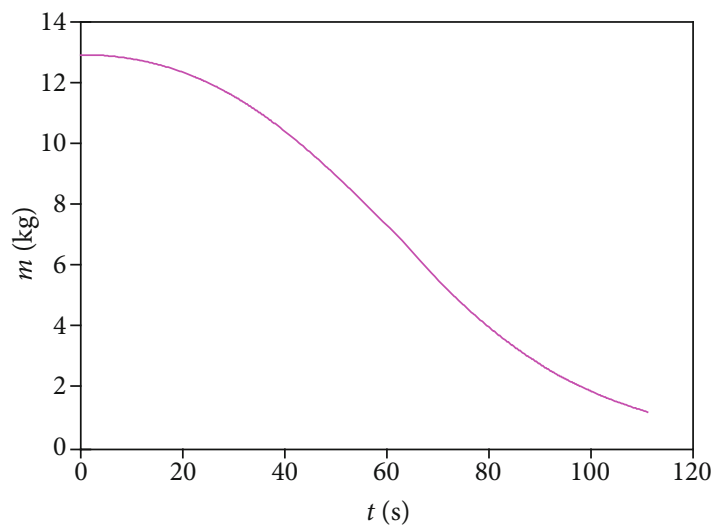

FIGURE 14: Curve of variation in the mass of air inside the large module over time.

For PROFILE, the law of variation in ambient pressure over time is written into a file and settings are made in pressure outlet boundary conditions. In the computation process, the program performs interpolation according to the data in the file to derive ambient pressure in each time step and update calculation. Therefore, this paper chooses PROFILE to set ambient pressure boundaries.

3.2.3. Time Step. When performing numerical simulation on the process of decompression in the module, time step is a key parameter that influences the accuracy of calculation. To meet the requirements of accuracy and quickness, the impact of time step on the results of calculation is explored. First, create a two-dimensional axisymmetric model for analysis. The volumes of the large/small modules, the sizes of the opening between the large/small modules, and the opening in the large module of this model are approximately the same as those of the original model. In the computation process, the initial pressure in the module is set to standard atmospheric pressure and ambient pressure is defined using the PROFILE file.

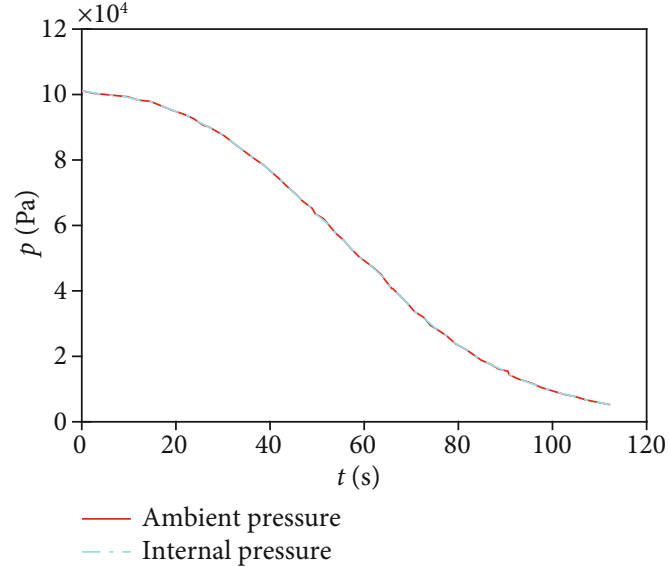

Figure 15: Curves of pressure inside the large module over time.

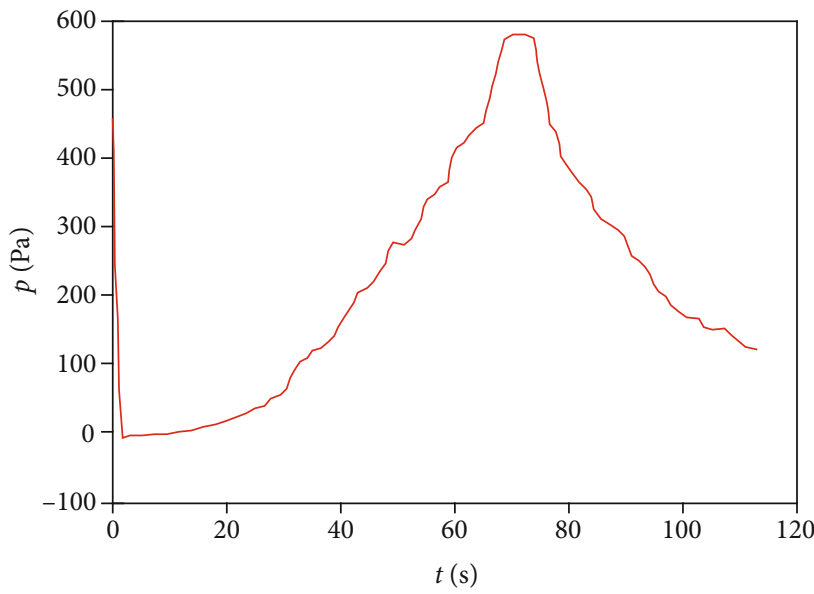

FIGURE 16: Curve of variation in pressure differential between the inside and outside of the wall of the large module over time.

Calculation is carried out with different time steps. The law of pressure variation in the module is shown in Figure 11. As shown in Figure 11(a), the pressure drop curves to which different time steps and the number of iterations correspond are overlapped after the flow state is created and the flow is stable. Therefore, the time steps and the number of iterations have less influence; however, as shown in Figure 11(b), the pressure curves which large time steps correspond fluctuate greatly in the process of creation of flow state in the initial stage.

Hence, smaller time steps are adopted to accurately simulate variation in velocity and pressure in the process of creation of flow state in the initial stage, with requirements of accuracy and quickness in mind; with the advancement of calculation time, time steps are gradually enlarged after the flow state is created.

Comparative analysis shows that pressure already changes slowly when the time step is $0.001 \mathrm{~s}$ during the creation of flow stage in the initial stage. After the creation of flow state, it is still necessary to consider quickness and accuracy when choosing the final time step. Therefore, a three- 


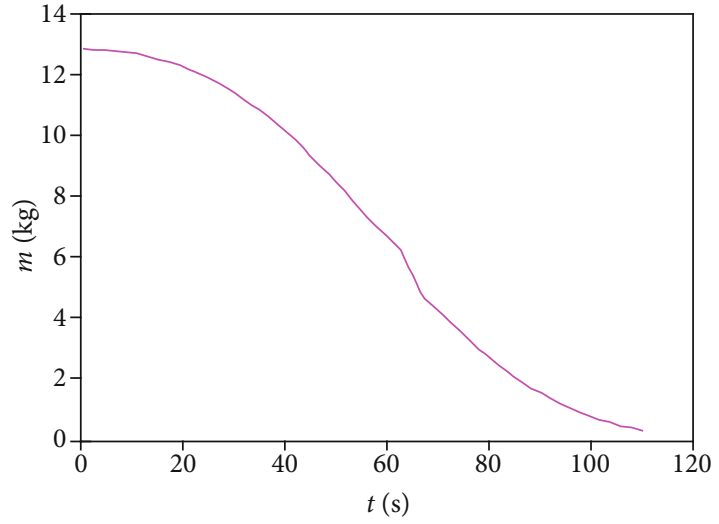

FIgURE 17: Curve of variation in the mass of air inside the large module over time.

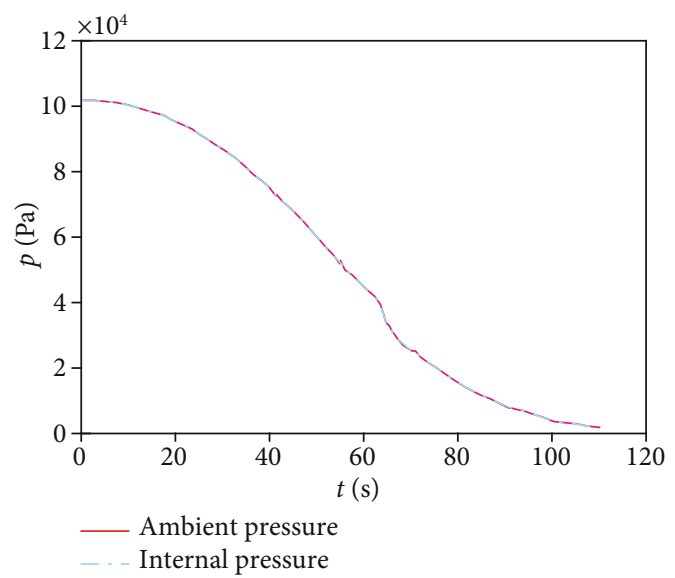

FIGURE 18: Curves of pressure in the large module over time.

dimensional computational model for the large/small modules is created and the final time steps of $0.01 \mathrm{~s}$ and $0.02 \mathrm{~s}$ are chosen. The ambient pressure is the lower limit of internal pressure in condition 1 , and numerical simulation is performed on variation of pressure inside the large/small modules.

The curves of variations in the ambient pressure and the pressure in the wall surface of the large module and the bottom surface and the sidewall surface of the small module are shown in Figure 12. The results show that pressure in the large/small modules declines as the ambient pressure drops; there is no significant difference of pressure in the large/ small modules when adopting different time steps.

The curves of pressure difference between the pressure in the large/small modules and the ambient pressure are shown in Figure 13. The results show that the differences between the intensities of pressure in the bottom and side of the large/small modules and the ambient pressure are almost consistent when the maximum time steps are the same; the differences between the intensities of pressure in various modules and the ambient pressure are comparatively consistent initially $(0-30 \mathrm{~s})$ when the maximum time steps are different; the gap between the pressure differentials for various modules to which different time steps correspond increases gradually when the ambient pressure decreases more quickly; the pressure differentials for various modules to

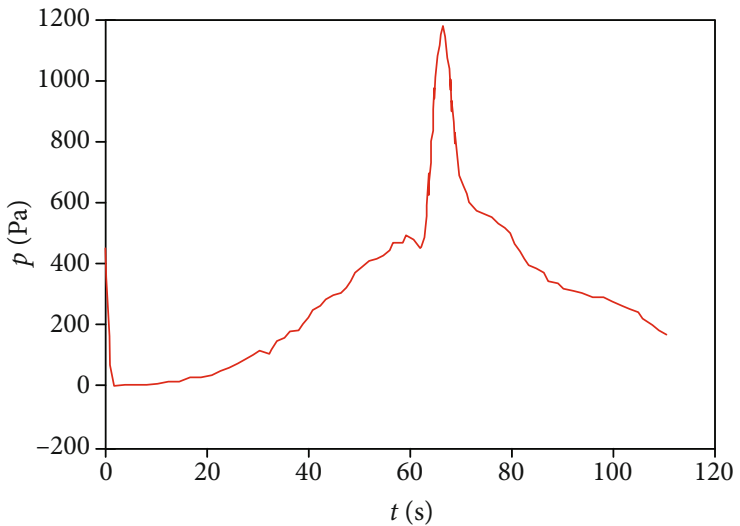

Figure 19: Curve of variation in pressure differential between the inside and outside of the large module over time.

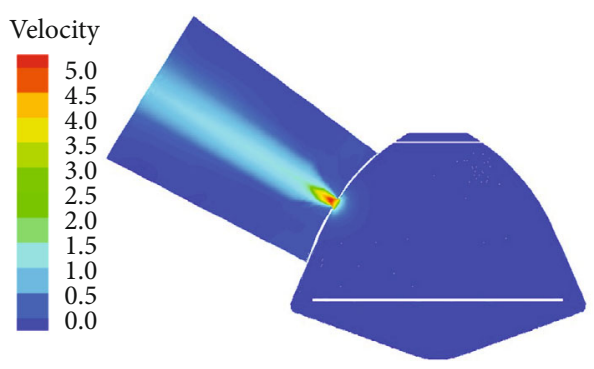

Figure 20: Velocity contour in the center section of the large and small modules at $t=18 \mathrm{~s}$.

which different maximum time steps correspond tend to be the same as the ambient pressure drops more slowly.

In summary, when the maximum time step selected is larger, the value of the difference between the intensities of pressure in various modules and the ambient pressure is relatively big and the results are more conservative. Therefore, we choose $0.02 \mathrm{~s}$ as maximum time step to meet the requirements of quickness and accuracy.

\section{Computational Results and Analysis}

Simulate the process of decompression in the module. First, simulate the decompression in the separate large module and analyze the variation characteristics of pressure in the large module when the ambient pressure is the upper and lower limits of internal pressure in condition 1; then, simultaneously considering the impact of the large/small modules, simulate the actual deflation process of the large/small modules and analyze the variation characteristics of pressure inside the large/small modules when the ambient pressure is the upper and lower limits of internal pressure in conditions 1 and 2 .

\subsection{Computation for the Separate Large Module}

4.1.1. The Upper Limit of Internal Pressure in Pressure Drop Condition 1 Is Selected as Ambient Pressure. When the upper limit of internal pressure in condition 1 is selected as 


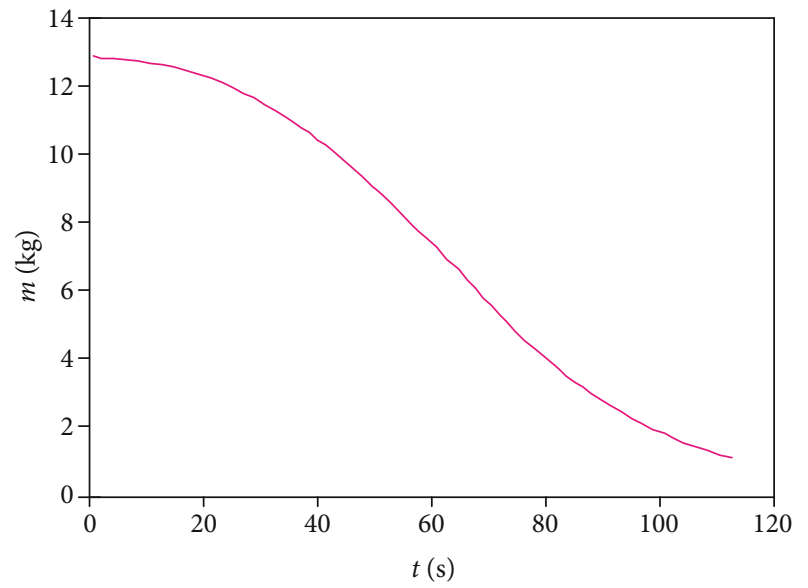

Figure 21: Curve of variation in the mass of air in the large module.

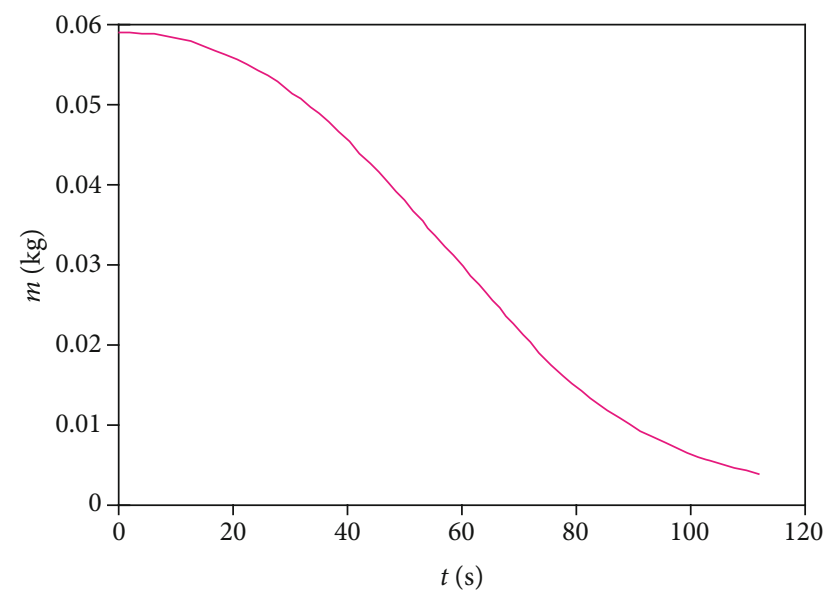

FIGURE 22: Curve of variation in the mass of air in the small module.

ambient pressure for pressure outlet boundary conditions, the curve of variation in the mass of gas in the large module over time is shown in Figure 14. The results show that the gas is gradually expelled from the module and the mass of gas inside the large module decreases gradually as the ambient pressure declines gradually.

Figure 15 shows the variation curve of wall pressure and environmental pressure in the large module over time. The pressure in the cabin decreases with the decrease of environmental pressure.

The curve of variation in the pressure differential between the inside and outside of the wall of the large module over time is shown in Figure 16. At the initial moment $(t=0 \mathrm{~s})$, the pressure inside the module is standard atmospheric pressure and differs greatly from ambient pressure, and in $1 \mathrm{~s}$, the pressure inside the module falls quickly and the pressure differential declines fast accordingly; subsequently, the environmental pressure gradually decreased, and the decline rate gradually increased; the cabin wall inside and outside the pressure difference gradually

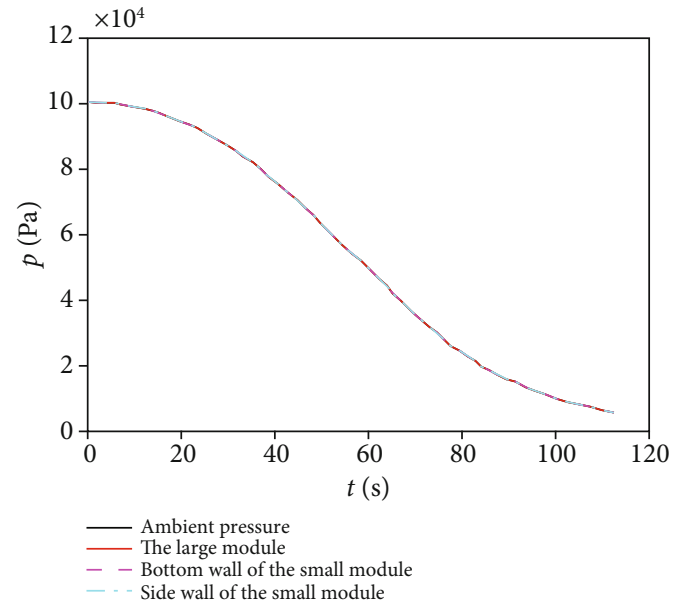

Figure 23: Curves of pressure in the wall surfaces of the large/small modules.

increased; at last, ambient pressure drops at a gradually decreasing speed and the pressure differential between the inside and outside of the wall surface of the module decreases gradually and accordingly again. The maximum value of the pressure differential between the inside and outside of the wall surface of the module is smaller than $600 \mathrm{~Pa}$.

4.1.2. The Lower Limit of Internal Pressure in Condition 1 Is Selected as Ambient Pressure. When the lower limit of internal pressure in condition 1 is selected as ambient pressure for pressure outlet boundary conditions, the curve of variation in the mass of gas inside the large module over time is shown in Figure 17. The gas is gradually expelled from the module, and the mass of gas inside the large module decreases gradually as the ambient pressure declines gradually.

The curves of pressure in the wall surface of the large module and the ambient pressure over time are shown in Figure 18. The pressure inside the module decreases as the ambient pressure decreases.

Figure 19 shows the curve of variation in the pressure differential between the inside and outside of the wall of the large module over time. The results suggest that, at the initial moment (0-60s), this curve is similar to the curve of variation in the pressure differential under the condition in which the upper limit of internal pressure is selected as ambient pressure in Section 4.1.1; after $60 \mathrm{~s}$, ambient pressure plunges, leading to a sudden increase in pressure differential between the inside and outside of the module; then, as the module continued to relieve pressure, the pressure difference gradually decreased. The maximum value of pressure differential between the inside and outside of the wall of the module does not exceed $1200 \mathrm{~Pa}$.

\subsection{Computation for the Large/Small Modules}

4.2.1. The Upper Limit of Internal Pressure in Pressure Drop Conditions Is Selected as Ambient Pressure. When the upper limit of internal pressure in condition 1 is selected as ambient pressure for pressure outlet boundary conditions, the 


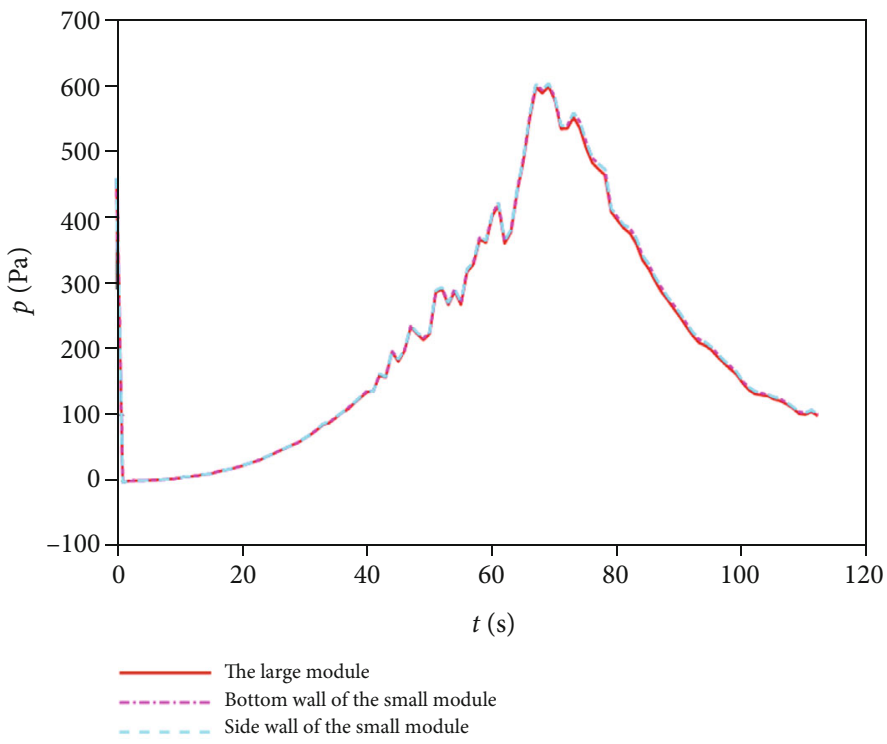

Figure 24: Curves of variation in pressure differential between the inside and outside of the large and small modules.

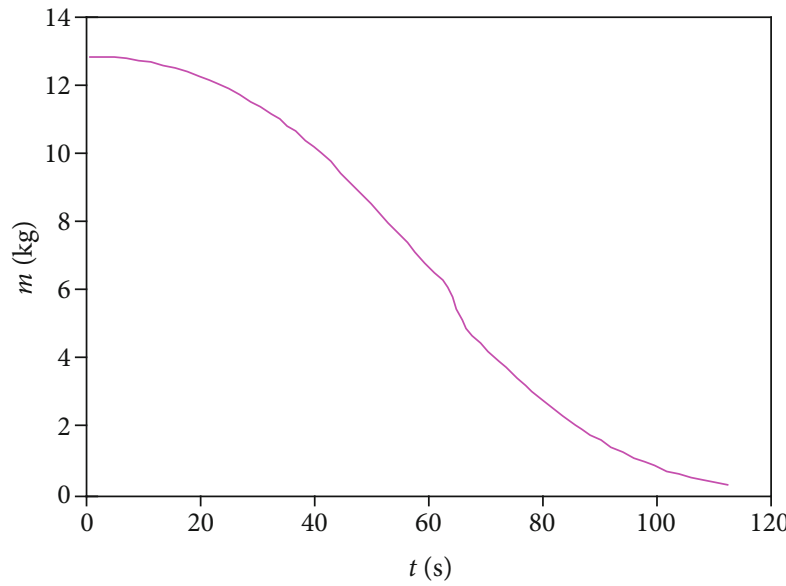

Figure 25: Curve of variation in the mass of air in the large module.

velocity contour in the center section of the large and small modules at $t=18 \mathrm{~s}$ is shown in Figure 20. The results show that the velocity increases gradually near the pressure relief hole, and when reaching the exit, the velocity increases sharply, and the maximum velocity is closed to Ma5, showing the outward jet shape, and then decreases gradually.

Figures 21 and 22 show the curves of variation in the mass of gases inside the large and small modules. The results show that the gas is expelled gradually from the module and the masses of gases inside the large/small modules decrease gradually as the ambient pressure declines gradually.

The curves of pressure in the inner wall surface of the large module, the bottom and side of the inner wall of the small module, and the ambient pressure over time are shown in Figure 23. It can be seen that the pressure inside the large/small modules is consistent and decreases as ambient pressure drops.

The curves of variation in pressure differential between the inside and outside of the inner wall surface of the large

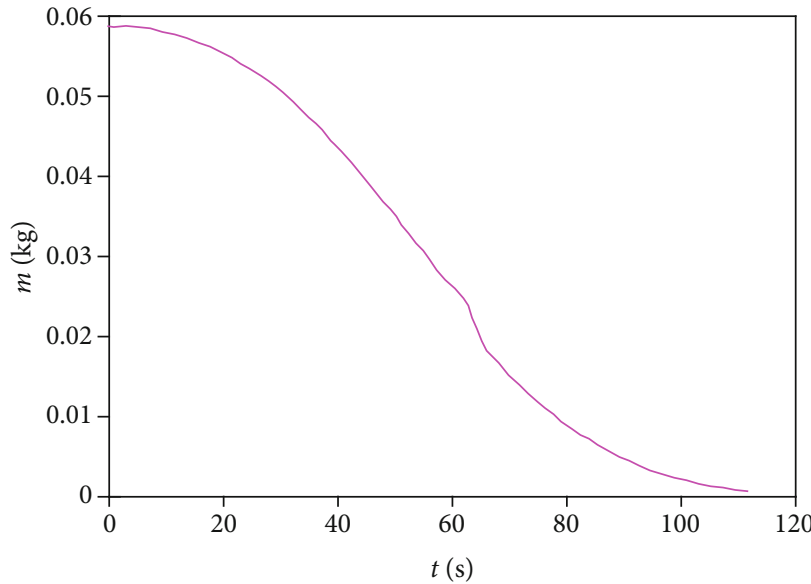

FIgURE 26: Curve of variation in the mass of air in the small module.

module and the bottom surface and the sidewall surface of the small module over time are shown in Figure 24. At the initial moment $(t=0 \mathrm{~s})$, the pressure inside the module is standard atmospheric pressure, which has a certain pressure difference from the ambient pressure; the pressure differential drops quickly as the module is deflated; thereafter, ambient pressure decreases gradually and at a gradually increasing speed, and the pressure differential between the inside and outside of the wall surface of the module increases gradually; over time, ambient pressure goes down at a gradually decreasing speed and the pressure differential between the inside and outside of the wall surface of the module declines gradually. The maximum value of the pressure differential between the inside and outside of the wall surface of the module is about $600 \mathrm{~Pa}$. Condition 1 Is Selected as Ambient Pressure. When the lower 


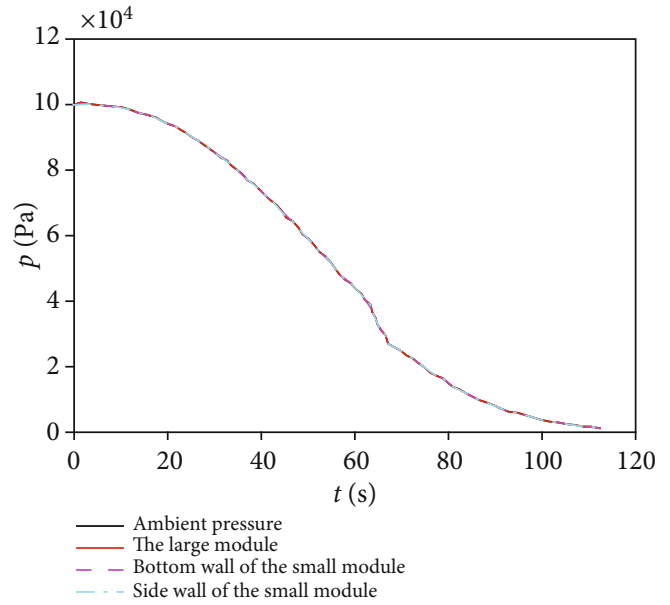

Figure 27: Curves of pressure in the wall surfaces of the large and small modules.

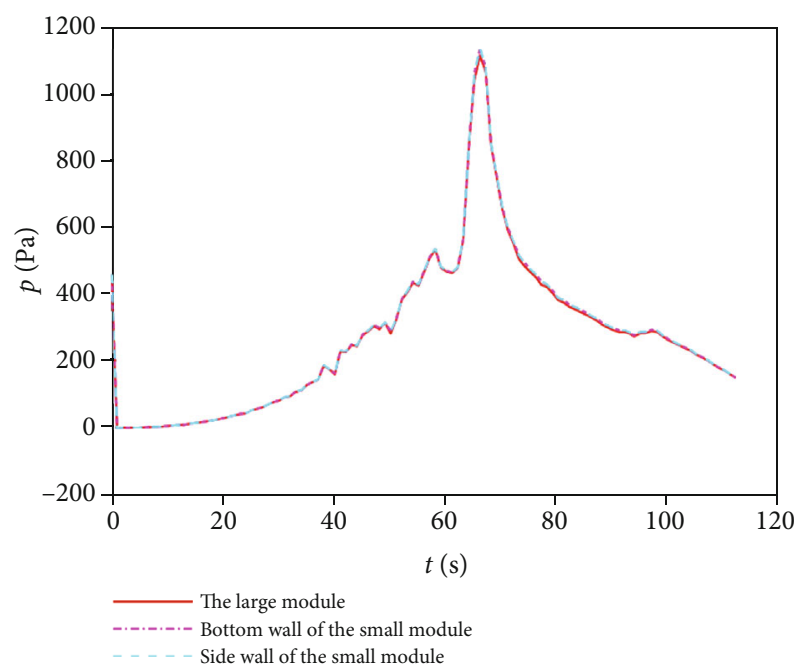

Figure 28: Curves of variation in pressure differential for the large and small modules.

limit of internal pressure in condition 1 is selected as ambient pressure for pressure outlet boundary conditions, the curves of variation in the mass of gas inside the large and small modules are shown in Figures 25 and 26. The results show that with the decrease of the ambient pressure, the gas in the module is gradually expelled, and the mass of the gas in both the large and small modules decreases gradually.

The curves of pressure in the inner wall surface of the large module, the bottom surface and the side of the inner wall of the small module, and the ambient pressure over time are shown in Figure 27. The pressure inside the large and small modules declines as ambient pressure drops.

The curves of variation in the pressure differential between the inside and outside of the inner wall surface of the large module and the bottom surface and the sidewall surface of the small module over time are shown in Figure 28. At the initial moment (0-62 s), the pressure differential between the inside and outside of the wall of the mod-

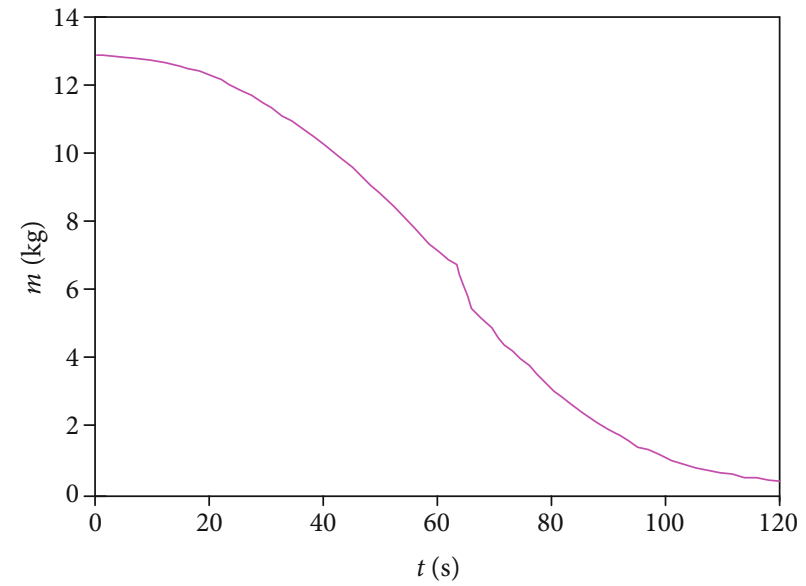

FIgURE 29: Curve of variation in the mass of air in the large module.

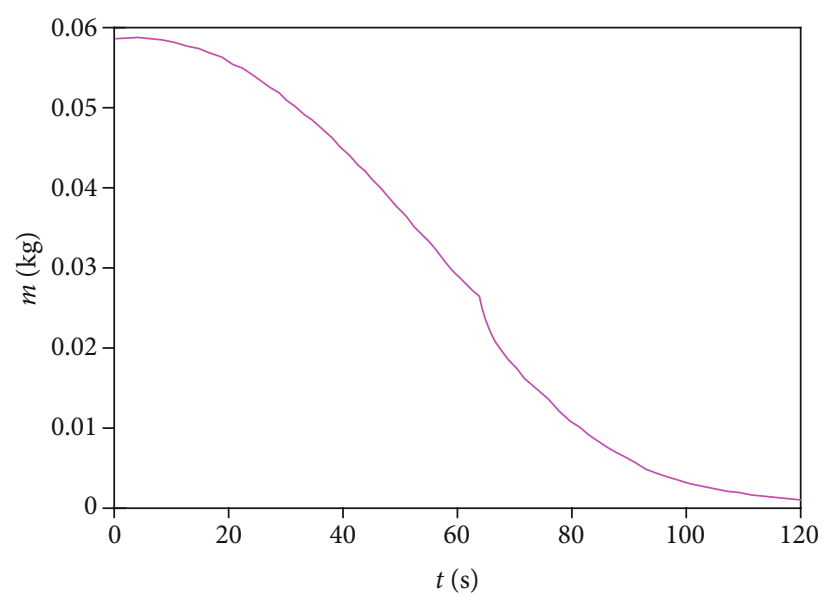

Figure 30: Curve of variation in the mass of air in the small module.

ule increases gradually over time; after $62 \mathrm{~s}$, the pressure differential reaches a brief peak due to an accelerated decline in ambient pressure; thereafter, the increase in the pressure differential causes a rise in the speed of decompression, thus leading to a quick drop in the pressure differential. The maximum value of the pressure differential is less than $1200 \mathrm{~Pa}$.

4.2.3. Pressure Drop Condition 2 Is Selected as Ambient Pressure. When pressure condition 2 is selected as ambient pressure in pressure outlet boundary conditions, the curves of variation in the mass of gas in the large/small modules are shown in Figures 29 and 30. The results suggest that the gas is expelled gradually from the module and the masses of gas inside the large/small modules decline gradually as the ambient pressure decreases gradually.

The curves of pressure in the inner wall surface of the large module, the bottom and the side of the inner wall of the small module, and the ambient pressure over time are shown in Figure 31. It can be seen that the pressure inside the large/small modules decreases as ambient pressure goes down. 


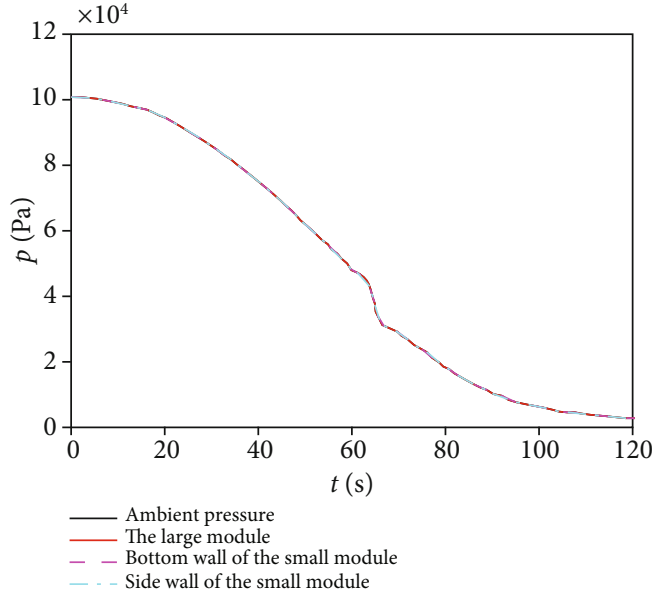

Figure 31: Curves of pressure in the wall surface of the large and small modules.

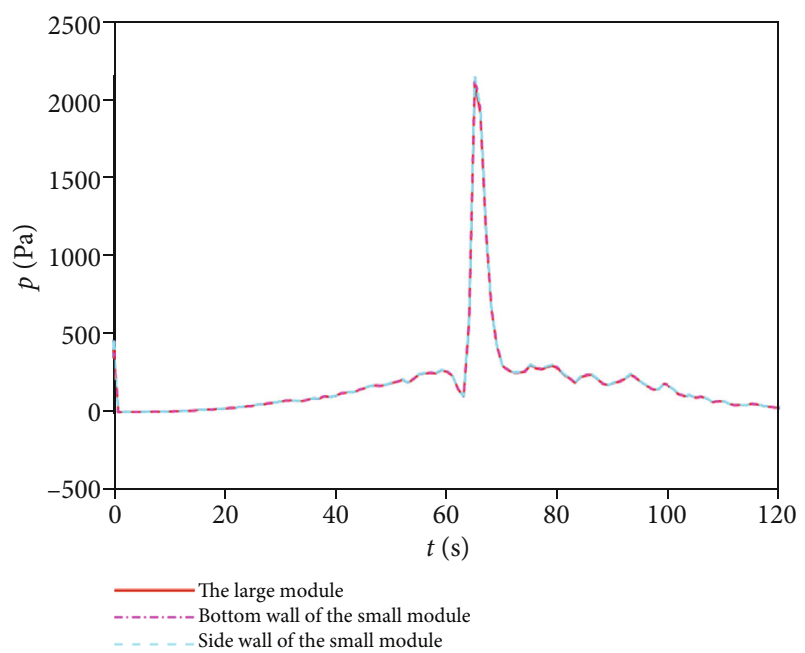

FIGURE 32: Curves of variation in the pressure differential between the inside and outside of the large module.

The curves of variation in the pressure differential between the inside and outside of the inner wall surface of the large module and the bottom surface and the sidewall surface of the small module over time are shown in Figure 32. At the initial moment $(t=0 \mathrm{~s})$, the pressure inside the module is standard atmospheric pressure, which has a certain pressure difference from the ambient pressure; the pressure differential drops quickly as the module is deflated; thereafter, ambient pressure goes down gradually and at a gradually increasing speed, and the pressure differential between the inside and outside of the wall surface of the module increases gradually and accordingly; over time, ambient pressure declines at a gradually decreasing speed, and the pressure differential between the inside and outside of the wall of the module falls gradually and accordingly. When $t=65 \mathrm{~s}$, ambient pressure drops dramatically to $6381 \mathrm{~Pa}$ in $1 \mathrm{~s}$ and the pressure differential between the inside and outside of the wall surface of the small module reaches a maximum, at around $2150 \mathrm{~Pa}$.

\section{Conclusion}

This paper uses FLUENT to perform numerical simulation on the decompression process of the Mars rover, develops outlet boundary conditions for PROFILE, and investigates the impact of ambient pressure settings, time steps, and the density of meshes on the results of simulation to improve the accuracy of computational results. The decompression process of the separate large module and the large/small modules under the ambient pressure of conditions 1 and 2 is simulated. The results show that when the ambient pressure is the upper limit and lower limit of internal pressure in condition 1 and condition 2 , respectively, the maximum internal and external pressure difference is less than $600 \mathrm{~Pa}, 1200 \mathrm{~Pa}$, and $2200 \mathrm{~Pa}$. Due to the small volume of the small module, the results for the separate large module and the large/small modules are basically consistent. The pressure differential between the inside and outside of the rover is mainly affected by changes in ambient pressure. In subsequent researches, on the one hand, the area of the opening should be increased and the following performance of pressure inside the module be sped up. On the other hand, the distortion of ambient pressure inside the fairing should be cut down to further reduce the pressure differential between the inside and outside of the rover.

\section{Data Availability}

The data used to support the findings of this study are available from the corresponding author upon request.

\section{Conflicts of Interest}

All authors declare no possible conflicts of interest.

\section{Authors' Contributions}

Wei Rao, Qi Li, and Rui Zhao participated in the research design. Weizhang Wang and Rui Zhao performed data analysis. Weizhang Wang and Hao Yan contributed to the writing of the manuscript.

\section{Acknowledgments}

The authors would like to acknowledge the support of the National Natural Science Foundation of China (Grant No. 11902025).

\section{References}

[1] J. Yingzi, Z. Zuchao, and Y. Qingjun, "Simplification and determination of polytropic exponent of THER- modynamic process in the filling and exhausting process in a pneumaitic system," Chinese Journal of Mechanical Engineering, vol. 41, no. 6, pp. 76-79, 2005.

[2] L. Jun, L. Yujun, and W. Zuwen, "Flow field calculation of pneumatic charging and discharging system," Machine Tool \& Hydraulics, vol. 2, pp. 24-26, 1999. 
[3] C. S. Landram, "Heat transfer during vessel discharge: mean and fluctuating gas temperature," Journal of Heat Transfer, vol. 95, no. 1, pp. 101-106, 1973.

[4] H. Zhanzhong, Z. Futang, and L. Yaofeng, "Numerical simulation of air flow in an engine inlet port," Vehicle \& Power Technology, vol. 2, pp. 49-53, 2001.

[5] Y. Lihong, Y. Qian, and L. Chengliang, "Study on measuring flow rate characteristics of pneumatic solenoid valves by isothermal chamber discharge," Mechanical Science and Technology, vol. 20, no. 10, pp. 1170-1172, 2005.

[6] J. C. Harley, Y. Huang, H. H. Bau, and J. N. Zemel, "Gas flow in micro-channels," Journal of Fluid Mechanics, vol. 284, pp. 257-274, 1995.

[7] D. Dongxing, T. Liyan, L. Zhixin, and G. Zengyuan, "Further research on the resistance characteristics of gas flow in micro tubes," Journal of Engineering Thermophysics, vol. 20, no. 5, pp. 603-607, 1999.

[8] J. Yingzi, Research on Condensation Of Pneumatic System, [Ph.D. thesis], Harbin Institute of Technology, 1998.

[9] L. Jun, Study on the Internal Condensation of Water Vapor in Pneumatic System, [Ph.D. thesis], Harbin Institute of Technology, 1999.

[10] J. Yingzi, L. Jun, B. Gang, and W. Zuwen, "Measurement and influence of propagation coefficient in the charging and releasing process in a pneumaitic system," Journal of Harbin Institute of Technology, vol. 30, no. 1, pp. 15-19, 1998.

[11] L. Chao, L. Hao, W. Fei, and Y. Xia, "A study of the outgassing characteristics of a vessel," Mechanical Science and Technology for Aerospace Engineering, vol. 1, 2011.

[12] L. Minghai, Z. Liqing, L. Chao, S. Guangmei, and C. Jun, "Analysis of pressure drop characteristics of deflation system under low atmospheric pressure environment," Acta Armamentarii, vol. 28, 2007.

[13] Y. Gang, X. Xiaowei, G. Longlong, and L. Baoren, "Characteristics of isovolumetric charging and releasing of high-pressure gas," Journal of Lanzhou University of Technology, vol. 36, no. 3, pp. 42-46, 2010.

[14] X. Huang Zhilong and Z. G. Dachuan, “Aerodynamic design and characteristic test of large intermittent wind tunnel control value," Journal of Experiments in Fluid Mechanics, vol. 26, no. 6, pp. 87-90, 2012.

[15] A. I. Kuptsov, R. R. Akberov, and F. M. Gimranov, "Calculation of gas parameters at the exit from a gas vent stack by means of calculating duration of emptying of the processing equipment," Contemporary Engineering Sciences, vol. 9, pp. 103-111, 2016. 